EPJ Web of Conferences 49, 03001 (2013)

DOI: 10.1051/epjconf/20134903001

(C) Owned by the authors, published by EDP Sciences, 2013

\title{
LHCb detector status and upgrade
}

\author{
O.Callot ${ }^{1}$ on behalf of the $\mathrm{LHCb}$ collaboration \\ ${ }^{1}$ Laboratoire de l'Accélérateur Linéaire, IN2P3/CNRS and Université Paris-Sud, Orsay, France
}

\begin{abstract}
The LHCb experiment is a specialized experiment for B and D physics at the CERN LHC. The layout of the experiment and the various components are described, with an overview of their performance. Global running conditions and performance are also shown. The motivation for the foreseen detector upgrade is presented, together with the proposed new detectors, to be installed during Long Shutdown 2, in 2018.
\end{abstract}

\section{General overview}

The $\mathrm{LHCb}$ detector is intended to study beauty and charm physics. At LHC, the production is mainly in the forward and backward directions. Both $\mathrm{b}$ particles are going in the same direction, thus a single arm spectrometer allows measuring the interesting $\mathrm{b}$ decay together with tracks from the other $b$ decay, for flavour tagging. The overall detector layout is shown in figure 1. The vertex locator measures all tracks near the interaction point, a dipole magnet and tracking stations after are providing accurate momentum measurement, two RICH detectors, calorimeters and a muon system provide full particle identification.

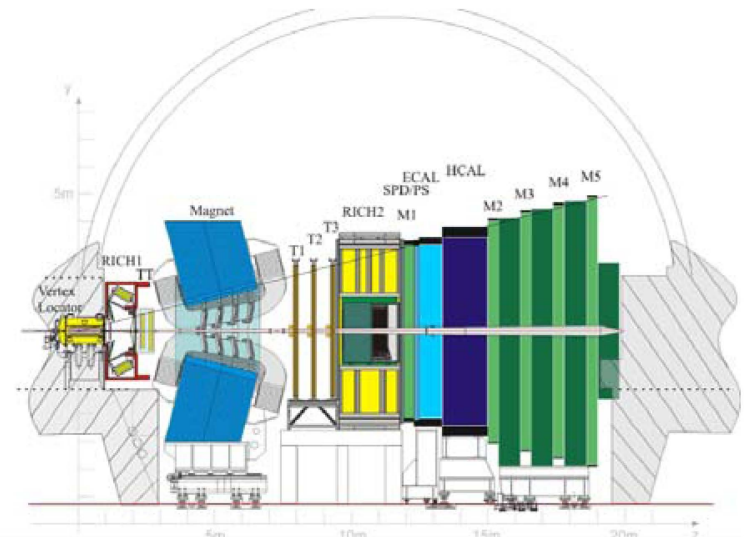

Fig. 1. The LHCb detector

\section{The various detector components}

\subsection{The Velo detector}

This detector located around the luminous region is made of 84 silicon micro-strips sensors, measuring either the radius or the azimuth of the hits. The closest radius is 8 $\mathrm{mm}$, and for that reason the detector is opened during machine injection, and closed only during "stable beam" physics data taking conditions. The mechanical position is reproducible to about 5 micrometres, and the beam position is stable within a fill as shown in figure 2 .

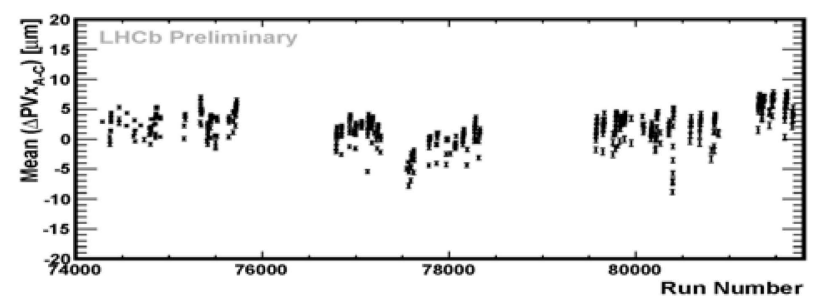

Fig. 2. Average distance between right-side primary vertices and left-side primary vertices.

The impact parameter resolution is essential to distinguish secondary tracks from primary tracks. A typical value is $20 \mu \mathrm{m}$ at $2 \mathrm{GeV} \mathrm{P}_{\mathrm{T}}$. The resolution is still slightly better on simulation, as shown in figure 3 . Work is in progress to understand it.

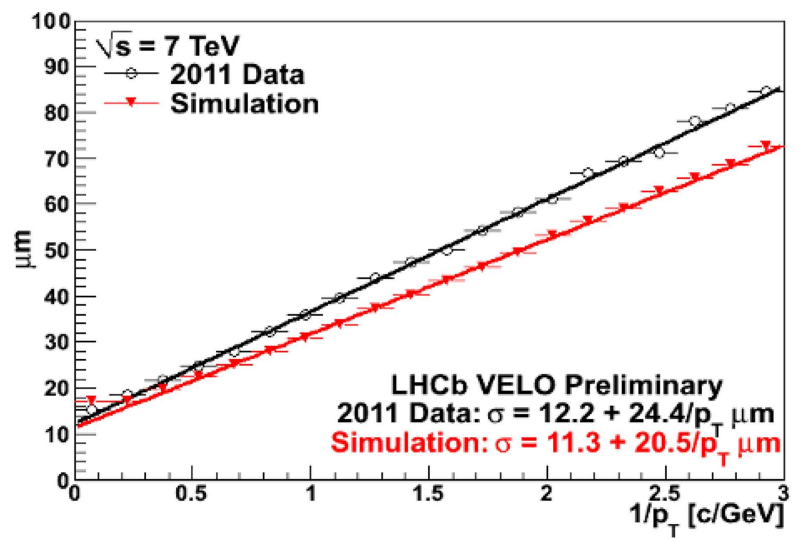

Fig. 3. Impact parameter resolution as function of $1 / \mathrm{P}_{\mathrm{T}}$. 
As the detector is close to the beam, it suffers radiation damages. The effect is visible on the dark current drawn by the sensors, and conforms to the prediction according to the delivered luminosity as shown on figure 4 .

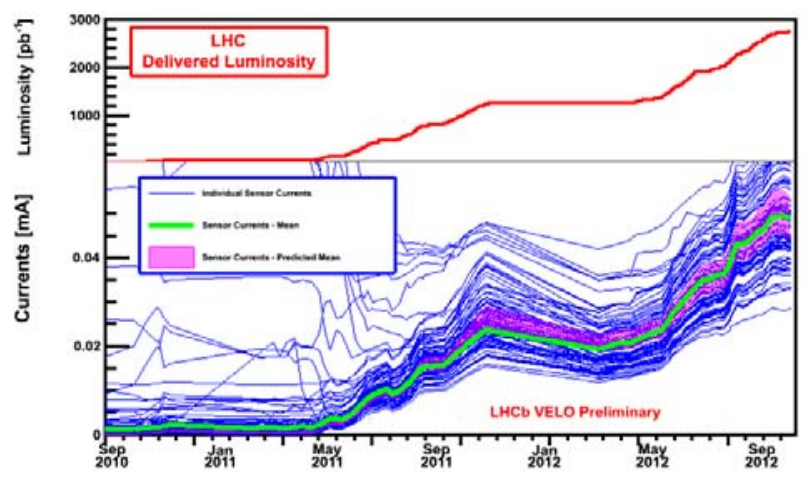

Fig. 4. Dark current of the Velo sensors versus time. The upper plot displays the LHC integrated luminosity.

\subsection{The tracking system}

One tracking station is situated before the magnet, called TT, consists of 4 layers of silicon strip detectors. Three stations of 4 layers each are situated after a 4 Tesla metre dipole, whose polarity is switched regularly to minimize acceptance systematics. Each station consists of $\mathrm{x}-\mathrm{u}-\mathrm{V}-\mathrm{X}$ arrangement, $x$ being the horizontal coordinate, where the tracks are bent by the magnet, $\mathrm{u}$ and $\mathrm{v}$ being layers with 5 degree stereo angle to measure the vertical coordinate.

The overall track reconstruction efficiency is over $96 \%$ for tracks over $5 \mathrm{GeV}$ of momentum.

\subsection{The RICH detectors}

These two detectors are identifying the particles from 2 to $100 \mathrm{GeV}$. One detector is located before the magnet with an aerogel and a C4F10 gas radiator, and one after the magnet with a CF4 gas radiator. The readout is performed by HPDs, about 500 tubes with 1024 channels each. Typically 10 HPDs are replaced each year due to degradation of their internal vacuum.

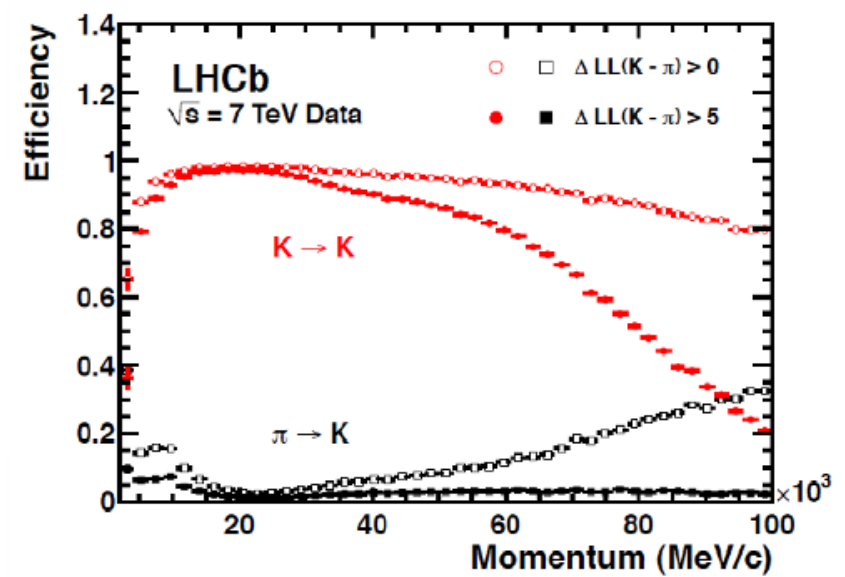

Fig. 5. Efficiency (circles) and contamination (squares) of kaon identification versus momentum for two different cuts.
The particle ID performance can be summarized as $95 \%$ efficiency for $5 \%$ contamination for the kaons coming from $\mathrm{B}$ decays. The figure 5 shows the efficiency and contamination for two values of the cuts, one for high efficiency and one for high purity.

\subsection{The calorimeter system}

This system has 4 components, from back to front: A hadronic calorimeter made of scintillating tiles inside an iron structure and readout by 1500 PMT with a resolution of $70 \% / \sqrt{E}+9 \%$ and is used essentially for the level- 0 trigger. The electromagnetic calorimeter is made of socalled "shashlik" blocks, a lead-scintillator stack readout by wave length shifting fibres and PTM. It has 6000 cells, and a resolution of $10 \% / \sqrt{\mathrm{E}}+1 \%$. The readout electronics is the same for these two detectors.

In front are two planes of scintillator tiles, with the same geometry as ECAL, separated by a lead converter. The first layer (SPD) identifies if the incoming particle is charged or neutral, and the second layer (PRS) if the shower started early, to identify electromagnetic showers.

\subsection{The muon system}

It consists of 5 stations interleaved with iron walls, the calorimeter system being the wall between the first two stations. It consists mainly of MWPC, with some GEM chambers for the central area of the first station. The geometry is projective, so that a muon originating from the luminous area and with infinite momentum crosses cells with the same number in the 5 stations.

The overall muon identification efficiency is about $97 \%$ with $3 \%$ miss-ID, mainly due to decay in flight. The layout of the system is shown on figure 6 .

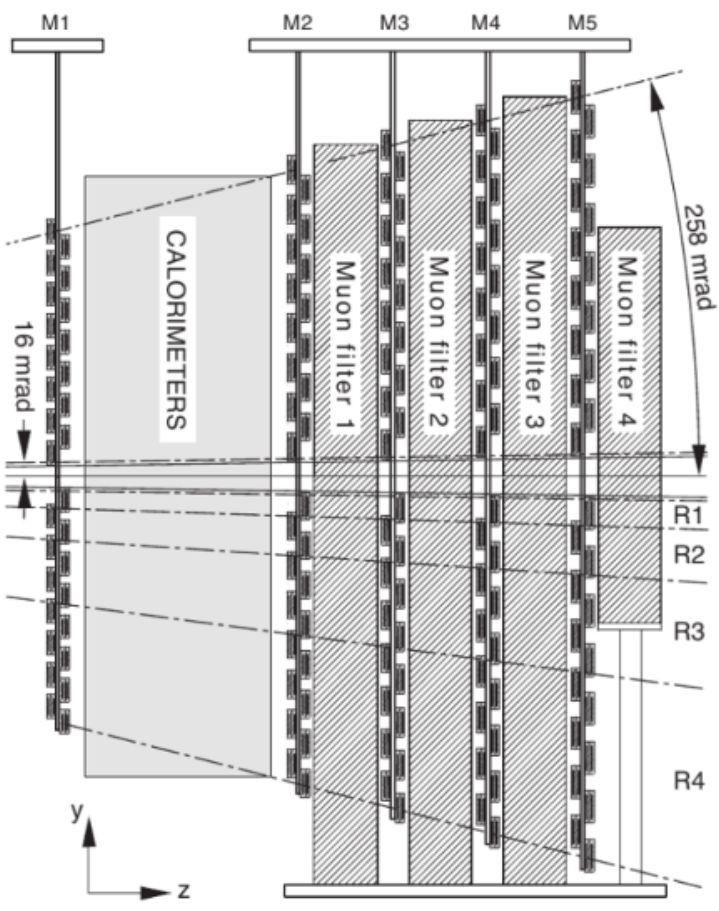

Fig. 6. Layout of the muon system 


\subsection{Trigger}

The first level (L0) is a hardware trigger, with custom electronics. It mainly selects events with high transverse momentum candidates, from clusters in the calorimeter (3.6 GeV for HCAL, 2.6 GeV for ECAL) or from tracks in the MUON system $(1.4 \mathrm{GeV})$. The accept rate is limited to $1 \mathrm{MHz}$, with a total latency of 4 microseconds.

The second level is a software trigger, running on a 1500 nodes farm, with about $30 \mathrm{k}$ copies of the code. It is logically a two-step process, first HLT1 selects events with a track having a large $\mathrm{P}_{\mathrm{T}}(1.6 \mathrm{GeV})$ and a large impact parameter (100 micrometres) as a signature of $\mathrm{B}$ decay products, then HLT2 runs the full reconstruction, without particle identification by the $\mathrm{RICH}$, and selects a large variety of physics channels in a semi-inclusive way. The total accepted rate is around $5 \mathrm{kHz}$.

\section{Running conditions}

\subsection{Luminosity}

$\mathrm{LHCb}$ was designed for single interaction crossings, as ambiguity in the assignment to a primary vertex was felt dangerous. A hardware multi-interaction veto was even built for that. But we found that it was possible to perform clean physics analysis with higher collision rate. The running conditions are currently 1.8 collisions per crossing, about 4 times higher than the design. Due to a mechanism called 'luminosity levelling', the luminosity is kept constant through the fill, the beams being kept slightly separated and moved closer from time to time to compensate for the decrease in beam intensities.

Figure 7 shows the LHC page1, on the left plot the decrease of the beam intensities, on the right the decrease of the luminosity for Atlas and CMS while the $\mathrm{LHCb}$ one is constant at a lower value. Figure 8 shows in more detail the LHCb luminosity, where the levelling steps are clearly visible, a few times per hour.

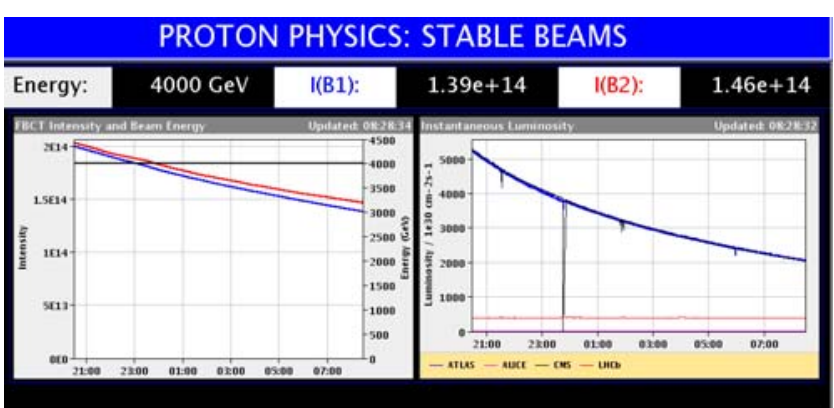

Fig. 7. LHC page1with beam intensities and luminosities

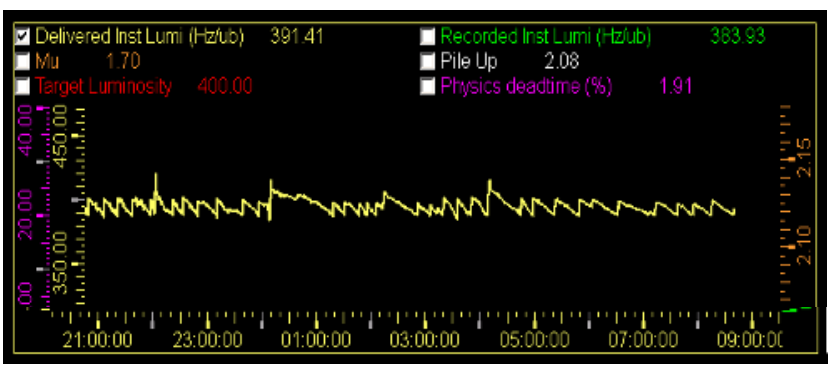

Fig. 8. LHCb luminosity, with the regular levelling.

\subsection{Operation performance}

The data taking efficiency is constantly monitored, to detect weaknesses in the system and fix them. The global value is $94.5 \%$ efficiency, the main contributors being $2.4 \%$ dead time, and $2.2 \%$ stops of the readout to recover the synchronisation of some systems.

\subsection{Reconstruction performance}

The momentum resolution is excellent, from $0.4 \%$ for $5 \mathrm{GeV}$ tracks to $0.6 \%$ at $100 \mathrm{GeV}$. This is important for studying narrow resonances as shown on figure 9 .

The particle identification is also working nicely, and depicted clearly on figure 10: The upper left plot is the two body mass plot assuming they are pions, while the other figures show the same plot when the particles are signed by the RICH system. The clear identification of the various modes, previously completely overlapping, is striking.
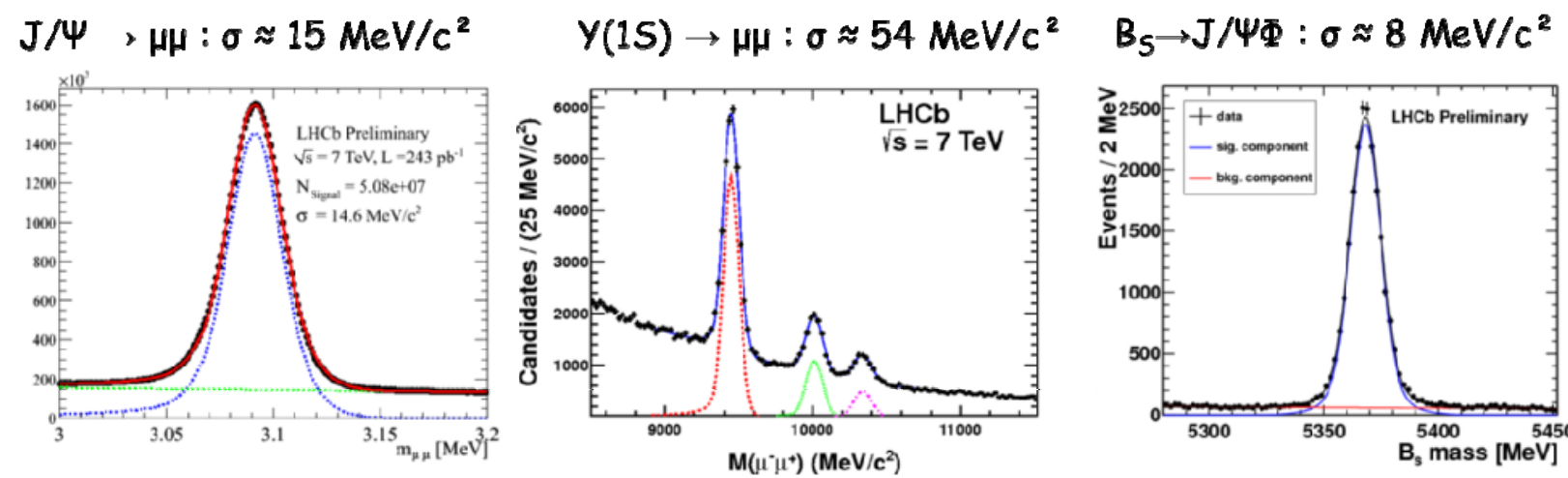

Fig. 9. Mass resolution for $\mathrm{J} / \Psi, \mathrm{Y}(1 \mathrm{~S})$ and $\mathrm{BS} \rightarrow \mathrm{J} / \Psi \Phi$. 


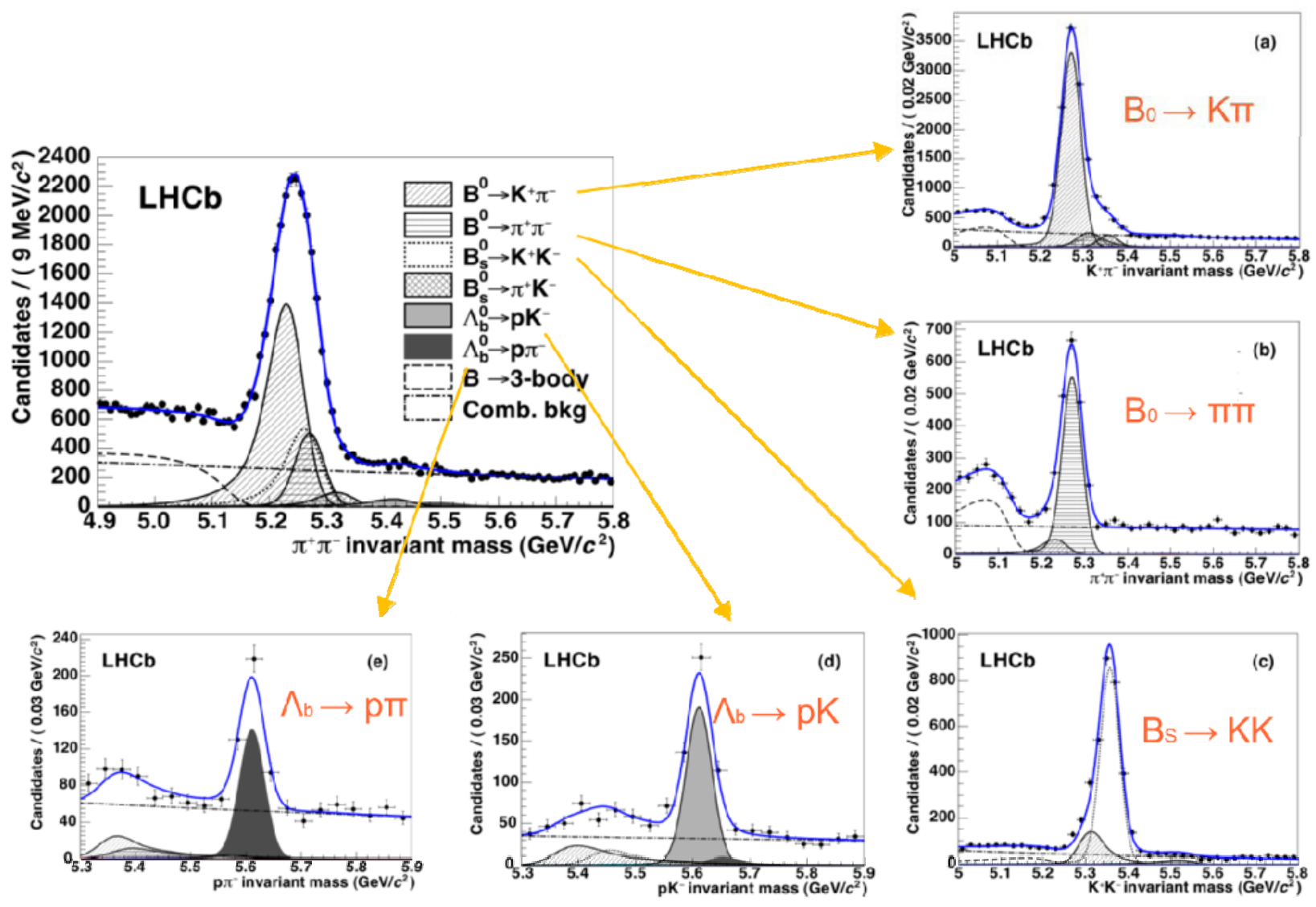

Fig. 10. Two body mass plot assuming pions (top left) or using particle identification

\section{The LHCb upgrade}

The current limitation of the detector is the L0 trigger, with the $1 \mathrm{MHz}$ hardware constraint. In order to stay within limits, the $\mathrm{P}_{\mathrm{T}}$ threshold for hadron has to be high, and about half of the interesting B decays are lost. The basic strategy for the upgrade, whose purpose is to increase the recorded luminosity with higher efficiency, is then to remove the L0 trigger. This implies reading out the detector at $40 \mathrm{MHz}$, and thus replacing all the frontend electronics. The second consequence is that the farm should process 40 times more events per second. Part of the increased performance will be obtained by faster processors, but detector choices will be influenced by the need for a faster software trigger. The target luminosity is initially to run at $1010^{32} \mathrm{~cm}^{-2} \mathrm{~s}^{-1}$ which is 2.5 times the current value with twice the number of bunches in the machine, only a slight increase in event complexity, but the detector should be able to run at twice that luminosity. Experience shows that we could handle more complex events.

\subsection{New vertex detector}

Two options are studied for replacing the current Velo. One option is to keep a similar layout of silicon microstrip detectors, with smaller pitch and $20 \%$ more channels per sensor. The layout of prototypes is shown on figure 11.

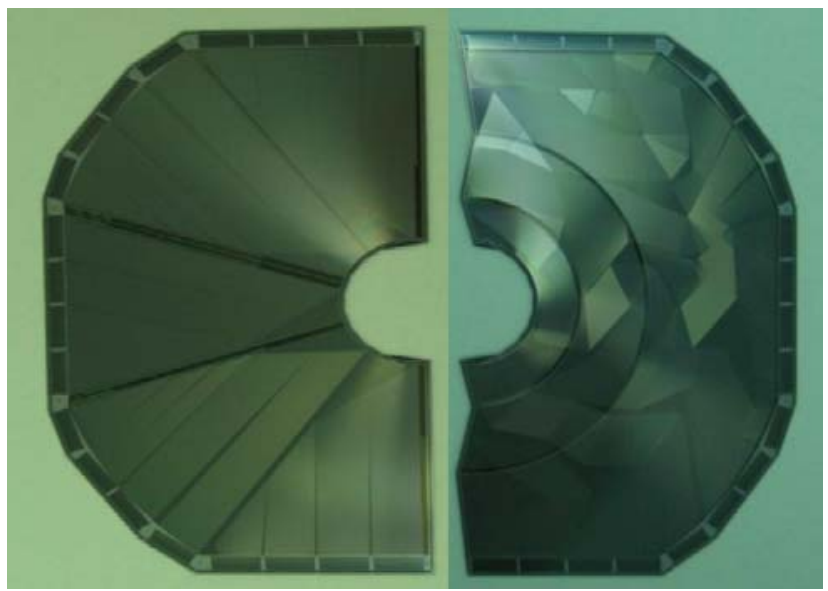

Fig. 11. Prototypes of the R (left) and Phi (right) sensors

The second option is a pixel detector, with square cells of 53 micrometres pitch. The whole detector will have about 40 million pixels, organised in 50 modules, 25 on each side.

\subsection{Tracking stations}

The tracking station in front of the magnet will be improved, still using the same silicon micro-strip 
technology. The goal is to guarantee a full coverage by overlapping sensors and getting closer to the beam pipe, while reducing the material in the acceptance. The idea is to require hits on this station, to make the momentum measurement faster in the trigger.

For the stations after the magnet, two options are studied. A conservative approach, similar to the current detector, with an Inner Tracker (silicon micro-strips) increased by a factor about 2 in sizes, so that the occupancy in the Outer Tracker (straw tubes) stays at an acceptable level. This is show on figure 12 .

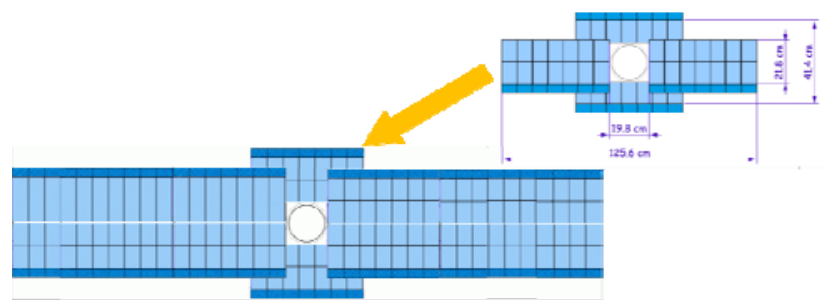

Fig. 12. Proposed layout of the new Inner Tracker. The top right part shows the current Inner Tracker.

The second option is to use scintillating fibres, 5 layers of $0.25 \mathrm{~mm}$ diameter as shown on figure 13 , with a fibre length of 2.5 meters to cover the whole acceptance, readout by silicon photo-multipliers. Intense $R \& D$ is ongoing to validate this option, mainly the radiation hardness of the SiPM and fibres and the mechanical accuracy on these large dimensions.

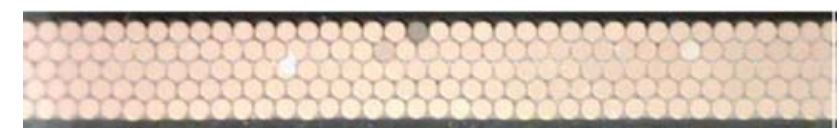

Fig. 13. Cross-section of the scintillating fibre plane

\subsection{The RICH}

The HPDs have to be replaced, and the main option is to use MAPMTs. This option was already studied when building $\mathrm{LHCb}$. The aerogel radiator will be removed, as it is not working well in very busy events. Studies are ongoing to improve the optics, to get a better resolution with less MAPMTs. More innovating ideas are also investigated, like the TORCH detector, a large time of flight detector with 15 ps resolution to identify the nature of low momentum particles. In view of the time and money constraints, this will only be for a future phase.

\subsection{Calorimeter}

The SPD and the PreShower will be removed as they will be too crowded. The electronics for the remaining calorimeters has to be adapted to the new readout scheme, and the gain of the PMs will be lowered to be less sensitive to aging, compensated by a higher gain in the electronics.

\subsection{Muon}

M1 will be removed, as its only use is for the L0 trigger. The electronics works already at $40 \mathrm{MHz}$ as it is used in the L0 trigger. As occupancy will increase, options are studied to replace the central part of M2 by GEM, and to build an improved electronics with less dead-time.

\subsection{Trigger}

As already mentioned, L0 will be suppressed. In fact a Low Level Trigger will be keep, re-using the L0 electronics, to minimize the load on the HLT farm if we can't have enough processing power at the beginning.

Implementing a very fast software trigger is a challenge that is addressed by various approaches. New trends in computing technology, like GPU or other multi core processors are investigated. The choice of the various candidates for new detector layout has the reconstruction speed as important ingredient. The software infrastructure and technology is being revisited. To process 30 times more events per second, we expect to gain a factor 10 by the progresses in the hardware provided by industry; we need to gain a factor 3 by improved detector design and software architecture.

\section{Summary}

$\mathrm{LHCb}$ is performing very well in difficult conditions, four times the number of collision per crossing than in the design. The detector is more than $99 \%$ operational, the quality of the reconstructed parameters is conform to the expectations. We collected so far a large statistics, with many results already published, and new results presented at this conference, mainly on 2011 data. Data taken in 2012 provide more than twice the statistics, and should lead to improved results in the forthcoming months. The preparation for the detector upgrade has started, and should provide 20 times more hadronic events per second from 2019 onwards. This is a very challenging, but realistic, project! 